\title{
Efeitos de um programa multiprofissional de tratamento da obesidade no ambiente aquático em adultos com obesidade severa
}

Effects of a multiprofessional program to treat obesity in the aquatic environment in adults with severe obesity

Efectos de un programa multiprofesional para tratar la obesidad en el ambiente acuático en adultos con obesidad grave

\author{
Mario Moreira Castilho \\ ORCID: https://orcid.org/0000-0002-4855-8236 \\ Universidade Estadual de Maringá, Brasil \\ E-mail: mmcastilho_1905@hotmail.com \\ Greice Westphal \\ ORCID: https://orcid.org/0000-0001-9107-0108 \\ Universidade Estadual de Maringá, Brasil \\ E-mail: greicewes@gmail.com \\ Regina Alves Thon \\ ORCID: https://orcid.org/0000-0003-2038-1640 \\ Universidade Estadual de Maringá, Brasil \\ E-mail: registhon@hotmail.com \\ Igor Alisson Spagnol Pereira \\ ORCID: https://orcid.org/0000-0001-7340-3909 \\ Universidade Estadual de Maringá, Brasil \\ E-mail: igorspagnol2@ hotmail.com \\ Fernando Malentaqui Martins \\ ORCID: https://orcid.org/0000-0003-1623-2183 \\ Universidade Estadual de Maringá, Brasil \\ E-mail: nandoesporte1@gmail.com \\ Michele Fernandes do Amaral \\ ORCID: https://orcid.org/0000-0002-4812-9773 \\ Universidade Estadual de Maringá, Brasil \\ E-mail: micheleamaralpsico@gmail.com \\ Rogério Toshiro Passos Okawa \\ ORCID: https://orcid.org/0000-0002-7116-274X \\ Universidade Estadual de Maringá, Brasil \\ E-mail: rpokawa@uem.br \\ Nelson Nardo Junior \\ ORCID: https://orcid.org/0000-0002-6862-7868 \\ Universidade Estadual de Maringá, Brasil \\ E-mail: nnjunior@uem.br
}

\begin{abstract}
Resumo
Introdução: $\mathrm{O}$ crescente aumento da obesidade severa e as comorbidades associadas a essa condição tornaram-se epidêmicas e atingem todas as idades e grupos econômicos. Além disso, as previsões são de que esse problema se torne ainda mais grave. Portanto, programas de tratamento da obesidade são urgentemente necessários, porém raramente são acessíveis à população de menor renda. Objetivo: Analisar os efeitos de um programa multiprofissional de tratamento da obesidade (PMTO) em adultos com obesidade severa (IMC $>40 \mathrm{~kg} / \mathrm{m}^{2}$ ). Método: Participaram do estudo 13 pessoas (seis mulheres e sete homens) com média de idade de 41,2 \pm 7 anos e IMC médio de $48,3 \mathrm{Kg} / \mathrm{m}^{2}$. Foram realizadas avaliações antropométricas, clínicas, laboratoriais, e de aptidão física, seguindo procedimentos padronizados, pré-intervenção e após 24 semanas de um PMTO. Resultados: O IMC, o percentual de (\%Gord.), gordura corporal absoluta, flexibilidade, força de preensão manual, força/resistência abdominal apresentaram melhoras significativas $(\mathrm{p}<0,05)$. Conclusão: O PMTO em adultos com obesidade severa mostrou-se efetivo na melhoria de vários parâmetros relacionados à saúde e de aptidão física, portanto, promissor para que mais estudos avaliem a sua aplicabilidade, incluindo intervenções no contexto da atenção primária à saúde (SUS).
\end{abstract}

Palavras-chave: Tratamento multiprofissional; Obesidade severa; Aptidão física. 


\begin{abstract}
Introduction: The continuous increase in severe obesity and the comorbidities associated with this condition have become epidemic and affect the people of all ages and economic groups. In addition, the predictions are that this problem will become even more serious. Therefore, obesity treatment programs are urgently needed, but are rarely accessible to the low-income population. Objective: To analyze the effects of a multiprofessional obesity treatment program (PMTO) in adults with severe obesity (BMI> $40 \mathrm{~kg} / \mathrm{m}^{2}$ ). Method: 13 people (six women and seven men) participated in the study, with a mean age of $41.2 \pm 7$ years and an average BMI of $48.3 \mathrm{~kg} / \mathrm{m}^{2}$. Anthropometric, clinical, laboratory, and physical fitness assessments were performed, following standardized procedures, preintervention and after 24 weeks of a PMTO. Results: BMI, percentage of fat (\% Fat), absolute body fat, flexibility, handgrip strength, abdominal strength/resistance showed significant improvements $(\mathrm{p}<0.05)$. Conclusion: PMTO in adults with severe obesity proved to be effective in improving several parameters related to health and physical fitness, therefore, promising for further studies to assess its applicability, including interventions in the context of primary health care (SUS).
\end{abstract}

Keywords: Multiprofessional treatment; Severe obesity; Physical fitness.

\title{
Resumen
}

Introducción: El aumento creciente de la obesidad severa y las comorbilidades asociadas a esta condición se han convertido en epidémicas y afectan a todas las edades y grupos económicos. Además, las predicciones apuntan a que este problema se agravará aún más. Por lo tanto, los programas de tratamiento de la obesidad se necesitan con urgencia, pero rara vez son accesibles a la población de bajos ingresos. Objetivo: Analizar los efectos de un programa multiprofesional de tratamiento de la obesidad (PMTO) en adultos con obesidad severa (IMC> $40 \mathrm{~kg} / \mathrm{m}^{2}$ ). Método: participaron del estudio 13 personas (seis mujeres y siete hombres), con una edad media de 41,2 \pm 7 años y un IMC medio de $48,3 \mathrm{~kg} / \mathrm{m}^{2}$. Se realizaron evaluaciones antropométricas, clínicas, de laboratorio y de aptitud física, siguiendo procedimientos estandarizados, antes de la intervención y después de 24 semanas de PMTO. Resultados: IMC, porcentaje de grasa (\% de grasa), grasa corporal absoluta, flexibilidad, fuerza de agarre, fuerza/resistencia abdominal mostraron mejoras significativas ( $\mathrm{p}<0.05$ ). Conclusión: PMTO en adultos con obesidad severa demostró ser eficaz en la mejora de varios parámetros relacionados con la salud y el estado físico, por lo tanto, prometedora para más estudios para evaluar su aplicabilidad, incluidas las intervenciones en el contexto de la atención primaria de salud (SUS).

Palabras clave: Tratamiento multiprofesional; Obesidad severa; Aptitud física.

\section{Introdução}

A obesidade é uma doença crônica de etiologia complexa e multifatorial, com elevado índice de impacto na saúde pública mundial (Abeso, 2020). A Organização Mundial da Saúde (OMS) estima que em 2025, cerca de 700 milhões de adultos estarão com obesidade (Abeso, 2016). Para o ano de 2025 a previsão é que a prevalência global da obesidade chegará a $18 \%$ nos homens e $21 \%$ nas mulheres e a obesidade grave chegará a $6 \%$ nos homens e $9 \%$ nas mulheres (Di Cesare et al., 2016).

No Brasil essa projeção já foi alcançada entre os homens, cuja prevalência atual ultrapassou os 18\%. Já entre as mulheres tudo indica que em breve o percentual será atingido (Brasil, 2016). Confirmando essa tendência, dado publicado recentemente pelo mesmo órgão aponta que a prevalência de obesidade continua aumentando no país, passando de $11,8 \%$ para 19,8\% entre os anos de 2006 e 2018 (Brasil, 2019). No ranking mundial da obesidade, o Brasil ocupa o terceiro lugar entre os homens, em um estudo que evolveu 200 países, ocupando o quinto lugar entre as mulheres. Já em relação à obesidade severa, o país ocupa o quinto lugar entre os homens e o quarto lugar entre as mulheres, (Di Cesare et al., 2016).

De forma global a obesidade é um dos problemas mais graves de saúde pública, pois além de afetar a saúde do indivíduo, causa consequências devastadoras na economia (Dobbs et al., 2014), estima-se que 7\% dos gastos com saúde destinam-se aos custos causados pela obesidade (Withrow \& Alter, 2011).

Para o tratamento da obesidade, a literatura apresenta como o procedimento básico (padrão) as intervenções multiprofissionais (terapia nutricional, exercício físico, tratamento psicológico e, se necessário, farmacológico), que direcione para mudanças comportamentais na alimentação e atividade física melhorando o estilo de vida ( Lau et al., 2007; Bevilaqua et al., 2016; Bianchini et al., 2016; Nardo Junior et al., 2018). A indicação de tratamento farmacológico ou cirúrgico depende de 
um acompanhamento clínico longitudinal desses pacientes e da ausência de respostas satisfatórias ao tratamento de base (Jensen et al., 2014; Lau et al., 2007; Stegenga et al., 2014).

Apesar do já descrito aumento da prevalência de obesidade grave, bem como de suas comorbidades. As formas de tratamento para essa doença costumam estar restritas a cirurgia bariátrica e farmacoterapia. Além disso, poucos ensaios clínicos têm avaliado os efeitos do tratamento multiprofissional da obesidade severa (Goodpaster et al., 2010).

Destaca-se que indivíduos com obesidade severa costumam apresentar risco elevado de comorbidades, baixa aptidão física, bem como limitações funcionais que prejudicam a autonomia para a realização de atividades rotineiras diárias (Pataky et al., 2014; Westphal et al., 2020)

Diante disso, o ambiente aquático torna-se apropriado para programas de condicionamento físico que integram as ações de equipes multidisciplinares. O exercício aquático reduz o impacto articular e o estresse térmico, sendo uma maneira eficaz de promoção de melhorias da capacidade funcional, aptidão cardiorrespiratória e qualidade de vida de pessoas com obesidade severa (Alberton \& Kruel, 2009; Zaniboni et al., 2019).

Considerando a necessidade de minimizar os danos que a obesidade severa causa as pessoas, este estudo teve por objetivo analisar os efeitos de um programa multiprofissional de tratamento da obesidade (PMTO) em adultos com obesidade severa.

\section{Metodologia}

Este estudo caracteriza-se como um ensaio clínico pragmático de intervenção (Patsopoulos, 2011). Os participantes foram convidados a partir de divulgação na mídia local (TV, rádio, jornal) e meios eletrônicos (site, e-mail institucional, Facebook) o qual buscava pessoas para a pesquisa, que atendessem aos seguintes critérios de inclusão: 1) ter idade entre 18 a 50 anos; 2) apresentar sobrepeso ou obesidade; 3) residir em Maringá, PR ou região metropolitana; 4) ter disponibilidade para participar integralmente das intervenções; 5) não ter sido submetido à cirurgia bariátrica; 6) não estar realizando outro tratamento para obesidade (terapias, medicamentos, exercícios físicos regulares); 7) atestado médico (liberação médica); e 8) assinar o Termo de Consentimento Livre e Esclarecido (TCLE).

Foram recrutados 358 participantes de um estudo maior que responderam as anamneses (dados sociodemográficos) e foram submetidos às avaliações preliminares (peso, altura, circunferência da cintura) durante 6 encontros entre os meses de dezembro/2017 a março/2018 realizados no Hospital Regional Universitário de Maringá (HUM). Destes, 252 participantes atenderam aos critérios de inclusão. Foram selecionados 30 participantes (16 mulheres e 14 homens) com obesidade severa (IMC $\geq 40 \mathrm{~kg} / \mathrm{m}^{2}$ ) selecionados para participar da pesquisa, que ocorreu no $1^{\circ}$ semestre de 2018 e teve duração de 24 semanas. Considerando somente aqueles que permaneceram até o final do programa, a amostra foi composta por 13 participantes, (seis mulheres e sete homens). Observa-se o procedimento de inclusão e amostra final na Figura 1. 
Figura 1. Fluxograma dos procedimentos de inclusão da amostra no programa.

\begin{tabular}{|c|}
\hline $\begin{array}{l}\text { Fase } 1 \text { - Avaliação do Risco Cardiometabólico. } \\
\text { Foram } 6 \text { encontros. } \\
\begin{array}{c}\text { Etapa } 1 \text { - Anamnese e avaliação preliminar. } \\
\text { n = } 358 \text { Participantes iniciais. }\end{array}\end{array}$ \\
\hline $\begin{array}{c}\text { Critérios de inclusão: } \\
1 \text { - Idade entre } 18 \text { e } 50 \text { anos; } \\
2 \text { - Ter sobrepeso ou obesidade; } \\
3 \text { - Residir em Maringá PR ou Regiảo Metropolitana; } \\
4 \text { - Disponibilidade para participar integralmente; } \\
5 \text { - Näo ter submetido à cirurgia bariátrica; } \\
\text { 6-Năo estar participando de outro tratamento para a obesidade; } \\
7 \text { - Atestado médico; } \\
8 \text { - Assinar o TCLE. }\end{array}$ \\
\hline $\begin{array}{l}\text { Etapa 2: Exames clínicos e laboratoriais. } \\
\qquad n=252 \text { elegiveis. }\end{array}$ \\
\hline $\begin{array}{l}\text { Não incluídos ou alocados em outros programas: } \\
\text {-Não atenderam aos critérios de inclusão; } \\
\text {-Restrição à prática de atividade em ambiente aquático. }\end{array}$ \\
\hline 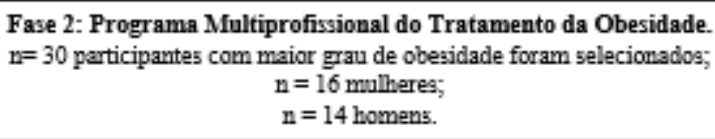 \\
\hline $\begin{array}{c}\mathbf{n}=17 \text { participantes desistiram no decorrer do programa: } \\
n=1 \text { por gravidez; } \\
n=5 \text { por trabalho; } \\
n=3 \text { por problemas de familia; } \\
n=5 \text { por transporte; } \\
n=3 \text { por horário. }\end{array}$ \\
\hline $\begin{array}{l}\text { Amostra final: } \\
n=13 \text { participantes; } \\
n=6 \text { mulheres; } \\
n=7 \text { homens; }\end{array}$ \\
\hline
\end{tabular}

Fonte: Autores.

Todos os procedimentos seguiram as regulamentações exigidas na Resolução 466/2012 do Conselho Nacional de Saúde para pesquisas envolvendo seres humanos. Os participantes leram e assinaram o Termo de Consentimento Livre e Esclarecido (TCLE), concordando em participar voluntariamente da pesquisa. O protocolo de pesquisa foi previamente aprovado pelo Comitê Permanente de Ética em Pesquisa da Universidade Estadual de Maringá (CAAE: 56721016.7.1001.0104, Parecer $n^{\circ}$ 2.655.268). Também foi submetido e aprovado pelo Registro Brasileiro de Ensaios Clínicos (ReBEC), plataforma do Ministério da Saúde, sob o registro RBR2yzs76.

Os participantes foram avaliados previamente por um médico cardiologista que a partir de uma anamnese, de exame físico e exames complementares (ecocardiograma, eletrocardiograma, e doppler de carótidas), atestou que os participantes estavam aptos a dar continuidade ao programa de avaliação física, na sequência no programa de intervenção proposto (PMTO).

Este PMTO teve duração de 24 semanas e foi composto por uma equipe multiprofissional com profissionais de Educação Física, Nutrição, Psicologia e Medicina. Foram realizados três encontros por semana, com duração de duas horas a cada encontro. Sendo que a primeira hora era destinada às intervenções teóricas das áreas da Educação Física, Nutrição e Psicologia, enquanto que a segunda hora foi destinada as intervenções práticas com exercícios em ambiente aquático.

Os dados foram coletados em dois momentos, pré-intervenção, na semana que antecipou o início do PMTO e pósintervenção, na semana subsequente ao término do PMTO. Os pesquisadores do NEMO das áreas de Educação Física, 
Nutrição e Psicologia realizaram as avaliações para coleta dos dados das variáveis antropométricas (peso, altura, circunferência do pescoço e da cintura); composição corporal (IMC, percentual de gordura e massa de gordura) e da aptidão física relacionada à saúde (flexibilidade, força de preensão manual, resistência muscular abdominal, resistência muscular membros inferiores e aptidão cardiorrespiratória).

Os parâmetros bioquímicos (glicemia, HbA1C, Insulina, Proteína C reativa, colesterol total e frações e triglicerídeos) foram coletados e analisados por profissionais habilitados em um laboratório público credenciado para essa finalidade e um laboratório comercial de referência localizado na cidade de Maringá, Paraná, com certificação ISO 9002 (International Organization for Standardization). As coletas de sangue para análise bioquímica foram realizadas sempre no período da manhã (entre 7 e 8 horas) com os pacientes em estado de jejum de no mínimo 8 a 10 horas.

Para avaliação das variáveis antropométricas e de composição corporal foram utilizados os seguintes instrumentos: um aparelho de bioimpedância elétrica, multifrequencial, octapolar, da marca Biospace (InBody®520) para medir o IMC, percentual de gordura e a massa de gordura; um estadiômetro de parede de 2,20 metros (Sanny®) para medir a altura; uma trena antropométrica flexível com resolução de 0,1 cm e amplitude de 2m (modelo Medical Starrett- SN- 51 4010, Sanny®) para medir as circunferências do pescoço $(\mathrm{CP})$ e da cintura $(\mathrm{CC})$. Para calcular o IMC utilizou-se a fórmula: peso $(\mathrm{kg}) /[$ altura (m) $\mathrm{x}$ altura $(\mathrm{m})]$.

Para avaliar as variáveis da aptidão física relacionada à saúde utilizaram-se os seguintes protocolos e materiais. A flexibilidade do tronco e dos músculos isquiotibiais foi mensurada através do teste de sentar e alcançar utilizando o banco de Wells. O procedimento foi realizado com o avaliado sentado e descalço, com os membros inferiores estendidos e os pés apoiados na superfície frontal do aparelho. A partir desta posição, flexionaram o tronco até o ponto máximo de seu alcance sobre o banco, no qual havia uma escalonada em centímetros utilizada para a avaliação desse parâmetro (Cardoso et al., 2007). Foram realizadas três tentativas, e considerou-se o maior valor obtido expresso em centímetros anotado no ponto mais distante alcançado com as pontas dos dedos. Foi utilizado um banco de Wells (modelo: BW2002, Sanny®) e um colchonete (ACSM, 2014).

Para avaliar a força muscular utilizou-se o teste de preensão manual, que mede a força máxima isométrica de preensão manual. O avaliado foi orientado a ficar na posição ortostática, segurar confortavelmente o dinamômetro com a mão dominante, ao longo do eixo longitudinal do corpo e ao comando exercer uma força máxima e breve. Foram realizadas três tentativas sendo adotada a maior medida, em quilogramas, apresentada pelo avaliado. Utilizou-se um dinamômetro modelo: GRIP D - TKK 5410, Takei® (Caputo et al., 2014).

Para avaliar a resistência muscular utilizou-se o teste de prancha abdominal, projetado para medir a resistência muscular estática da região do tronco, que envolve os músculos da região abdominal, lombar e pélvica. $\mathrm{O}$ avaliado foi orientado a se posicionar em decúbito ventral, apoiando apenas os antebraços e os dedos do pé no chão. Os cotovelos flexionados em $90^{\circ}$ afastados na largura dos ombros, pés alinhados com a largura do quadril, tornozelos em $90^{\circ}$, joelhos estendidos, quadril elevado numa posição reta com as costas e as mãos não podendo ficar juntas. Após assumir essa postura o avaliado permaneceu o máximo de tempo possível na posição. O teste é encerrado quando o participante desfaz a postura devido à fadiga ou interrompe voluntariamente o teste. Realizou-se uma única tentativa cujo resultado foi mensurado em segundos. Foram utilizados um colchonete e um cronômetro para a realização desse teste (Chase et al., 2014)

Para mensurar a resistência muscular dinâmica dos membros inferiores utilizou-se o teste de sentar e levantar. O avaliado iniciou o teste sentado em uma cadeira com as costas apoiadas, pés no chão e braços cruzados na região peitoral. Ao sinal, o avaliado ficou em pé e então retornou à posição sentada, devendo tentar realizar o maior número possível desse movimento, durante 30 segundos. Para este teste utilizou-se uma cadeira com encosto e sem braços, com altura de aproximadamente $43 \mathrm{~cm}$ e um cronômetro (Jones \& Rikli, 2012). 
Para avaliação da aptidão cardiorrespiratória utilizou-se o teste de caminhada de 6 minutos (TC6M), no qual o avaliado caminhou o mais rápido possível (sem correr) durante 6 minutos em um percurso de 20 metros demarcado com cones e trena. (ATS, 2002). Para a realização do TC6M foram utilizados os seguintes materiais: 6 cones pequenos, 1 trena de 50 metros, 1 fita adesiva, 1 cronometro (modelo: Casio Hs-3 digital), 1 escala de percepção de esforço, 2 cardiofrequencímetro (Polar modelo RS800CX), 1 prancheta. Ao sinal indicativo do avaliador, o participante caminhou o mais rápido possível (sem correr) em volta do percurso quantas vezes conseguisse, desde que dentro do limite de tempo. O participante foi instruído sobre a possibilidade de parar e descansar durante o teste, se necessário, e depois voltar a caminhar. $\mathrm{O}$ avaliador manteve contato visual com o avaliado para informar o tempo transcorrido e estimular o avaliado com frases padronizadas a cada 30 segundos. $\mathrm{O}$ avaliador informou o tempo restante de 3 minutos, 2 minutos e 1 minuto. Ao sinal de pare o avaliado foi orientado a parar no local e aguardar até que fosse anotada a distância percorrida. Além disso, a frequência cardíaca foi anotada antes do início do teste em repouso (FC_pré), imediatamente após o teste (FC_pós) e um minuto após a interrupção do teste (FC_recup.). A percepção subjetiva de esforço foi anotada após a execução do teste usando a escala adaptada de BORG (1982) (de 1 a 10).

Para a classificação do Índice de Massa Corporal (IMC) e a mensuração das circunferências do pescoço (CP) e da cintura (CC) utilizou-se os pontos de corte propostos pela Organização Mundial de Saúde (2011). Enquanto que as circunferências maiores ou iguais a $94 \mathrm{~cm}$ para homens e $>80 \mathrm{~cm}$ para mulheres representam risco de complicações metabólicas aumentado, sendo que quando esses valores são $>102 \mathrm{~cm}$ para homens e $>88 \mathrm{~cm}$ para mulheres a classificação é de risco substancialmente aumentado (WHO, 2011).

A classificação da glicemia em jejum seguiu os critérios da Diretriz da Sociedade Brasileira de Diabetes 2017-2018, que estabelece os seguintes valores para o diagnóstico: glicemia em jejum $<100 \mathrm{mg} / \mathrm{dl}$ normoglicemia; $\geq 100 \mathrm{a}<126 \mathrm{mg} / \mathrm{dl}$ pré-diabetes e $\geq 126 \mathrm{mg} / \mathrm{dL}$ diabetes estabelecido (Egídio et al., 2017).

Os parâmetros do perfil lipídico foram classificados de acordo com a Diretriz Brasileira de Dislipidemias e Prevenção da Aterosclerose 2017, que estabelece como nível elevado os triglicerídeos em jejum $\geq 150 \mathrm{mg} / \mathrm{dL}$ e colesterol total $\geq 190$ $\mathrm{mg} / \mathrm{dL}$ e nível baixo o HDL-c em jejum < $40 \mathrm{mg} / \mathrm{dL}$ para homens e < que $50 \mathrm{mg} / \mathrm{dL}$ para mulheres (Faludi et al., 2017).

As intervenções práticas com exercícios em ambiente aquático foram realizadas em uma piscina da universidade, com dimensões de $25 \times 12$ metros, profundidade de 1,40 metros, separadas por 6 raias, a temperatura foi mantida entre $29^{\circ}$ e $31^{\circ}$ graus e o potencial hidrogeniônico permaneceu entre 7 a 8 pH.

As aulas foram ministradas por dois profissionais de Educação Física do grupo NEMO. Esses profissionais realizaram, predominantemente, atividades de hidroginástica, também utilizando-se de exercícios de natação, com intensidade moderada, aumentada gradativamente ao longo do programa (Lopera et al., 2016). O programa foi composto por sessões de caminhada e alongamentos, com duração de 10 minutos, seguido da fase principal com duração de quarenta minutos e desaceleração (volta a calma) nos 10 minutos finais, conforme recomendações do ACSM (ACSM, 2014).

A percepção subjetiva de esforço (PSE) (Borg, 1982), foi utilizada para monitorar a intensidade das atividades. Assim, os participantes informaram a PSE em momentos pré-definidos e ao final das sessões.

Para o processamento e análise dos dados foram utilizados uma planilha do programa Microsoft Excel 2020 e as análises estatísticas foram realizadas por meio do pacote estatístico SPSS versão 20.0 (Andy Field, 2009). Para a normalidade dos dados utilizou-se o teste de Shapiro-Wilk. As variáveis foram apresentadas por: Média e Desvio-Padrão apresentando dados paramétricos e o teste $\mathrm{t}$ de student foi utilizado para verificar o efeito do PMTO (momento pré vs pós), o nível de significância adotado foi de $5 \%(\mathrm{p}<0,05)$. 


\section{Resultados}

Foram avaliados 13 participantes (6 mulheres e 7 homens) com média de idade de 41,2 \pm 7 anos. Na Tabela 1 estão apresentados os dados das variáveis antropométricas e de composição corporal do grupo nos momentos pré e pós intervenção.

Tabela 1. Análise dos efeitos da Intervenção Multiprofissional nas variáveis antropométricas e composição corporal após 24 semanas de intervenção.

\begin{tabular}{cccc}
\hline Variáveis & Pré-Intervenção & $\begin{array}{c}\text { Avaliação final } \\
\text { (24 Semanas) }\end{array}$ & Valor de p \\
\hline IMC $\left(\mathrm{Kg} / \mathrm{m}^{2}\right)$ & $48,3 \pm 6,3$ & $46,8 \pm 6,3$ & $\mathbf{0 , 0 4 5 6 *}$ \\
CP $(\mathrm{cm})$ & $44,2 \pm 3,6$ & $42,6 \pm 4,5$ & 0,5147 \\
CC $(\mathrm{cm})$ & $123,6 \pm 7,7$ & $122 \pm 8,5$ & 0,4446 \\
Percentual de Gordura $(\%)$ & $51,1 \pm 3,2$ & $50 \pm 4$ & $\mathbf{0 , 0 1 7 4} *$ \\
Massa de Gordura $(\%)$ & $72,4 \pm 13,2$ & $68,7 \pm 12,9$ & $\mathbf{0 , 0 1 5 9 *}$ \\
\hline
\end{tabular}

IMC: Índice de Massa Corporal. CP: Circunferência do Pescoço. CC: Circunferência da cintura.

*Teste t pareado significativo considerando nível de significância de 5\%. Fonte: Autores.

Observa-se na Tabela 1 que houve uma diminuição significativa no IMC ( $\mathrm{p}=0,04)$, no Percentual de Gordura. $(\mathrm{p}=0,01)$ e na Massa de Gordura $(\mathrm{p}<0,05)$. Apesar disso não foram verificadas alterações nas variáveis CP e CC.

Na Tabela 2 são apresentados os resultados das variáveis bioquímicas obtidos nos momentos pré e pós intervenção.

Tabela 2. Análise dos efeitos da Intervenção Multiprofissional nas variáveis bioquímicas após 24 semanas de intervenção.

\begin{tabular}{lccc}
\multicolumn{1}{c}{ Variáveis } & Pré-Intervenção & $\begin{array}{c}\text { Avaliação final } \\
\text { (24 Semanas) }\end{array}$ & $\begin{array}{c}\text { Valor de } \\
\text { p }\end{array}$ \\
\hline Glicemia (mg/dL) & & $105,9 \pm 13,7$ & 0,4755 \\
HbA1C (\%) & $103,5 \pm 10$ & $5,3 \pm 0.6$ & 0,0829 \\
Insulina (mU/L) & $5,5 \pm 0,6$ & $20,4 \pm 10$ & 0,0995 \\
PCR (mg/L) & $24,7 \pm 12,9$ & $5,5 \pm 4$ & 0,0869 \\
Colesterol Total(mg/dL) & $7,5 \pm 3,1$ & $183,3 \pm 29,4$ & 0,3454 \\
Triglicerídeos (mg/dL) & $196,6 \pm 39,9$ & $122,9 \pm 32,5$ & 0,1167 \\
\hline
\end{tabular}

HbA1C: Hemoglobina Glicada. PCR: Proteína C Reativa. *Teste t pareado significativo considerando nível de significância de 5\%. Fonte: Autores.

A Tabela 2 demonstra que entre os parâmetros bioquímicos não houve alterações significativas. Porém, em alguns casos, pode-se considerar que houve uma tendência a redução ( $\mathrm{p}<0,1)$ na $\mathrm{HbA} 1 \mathrm{C}$, Insulina, Proteína $\mathrm{C}$ reativa.

Na Tabela 3 estão apresentados os dados das variáveis de aptidão física relacionadas a saúde nos momentos pré e pós intervenção. 
Tabela 3. Análise dos efeitos da Intervenção Multiprofissional na aptidão física.

\begin{tabular}{|c|c|c|c|}
\hline Variáveis & Pré-Intervenção & $\begin{array}{l}\text { Avaliação } \\
\text { final } \\
(24 \\
\text { Semanas }) \\
\end{array}$ & $\begin{array}{c}\text { Valor de } \\
\text { p }\end{array}$ \\
\hline Flexibilidade $(\mathrm{cm})$ & $9,8 \pm 7,9$ & $13,9 \pm 9,3$ & $0,0037 *$ \\
\hline Força de preensão manual $(\mathrm{kg})$ & $35,3 \pm 11,2$ & $39,2 \pm 13,3$ & $\mathbf{0 , 0 1 3 1 *}$ \\
\hline Resistência muscular abdominal estática (seg) & $37,2 \pm 15,9$ & $49,5 \pm 19,8$ & $0,0064 *$ \\
\hline Resistência muscular membros inferiores dinâmica (n) & $13,3 \pm 3$ & $14,6 \pm 1,2$ & 0,0776 \\
\hline Freq. Cardíaca pré-teste em repouso (bpm) & $127,3 \pm 15,2$ & $120,9 \pm 13$ & 0,064 \\
\hline TC6M (distância percorrida em metros) & $500,6 \pm 56,9$ & $517,6 \pm 65,5$ & 0,8068 \\
\hline Freq. Cardíaca pós-teste (bpm) & $176,2 \pm 23,4$ & $172,8 \pm 24,3$ & 0,582 \\
\hline Freq. Cardíaca de Recup. 1min. Pós-teste (bpm) & $127,7 \pm 11,7$ & $121 \pm 20,7$ & 0,9721 \\
\hline PSE (Escala de Borg adaptada) & $4,3 \pm 2,1$ & $5,2 \pm 2$ & 0,1823 \\
\hline
\end{tabular}

Freq. Cardíaca pré-teste em repouso: Frequência Cardíaca pré-teste em repouso. TC6M: Teste de Caminhada de seis minutos. Freq. Cardíaca pós-teste: Frequência Cardíaca pós-teste. PSE: Percepção Subjetiva de Esforço. *Teste t pareado significativo considerando nível de significância de 5\%. Fonte: Autores.

De acordo com a Tabela 3, houve melhora estatisticamente significativa $(\mathrm{p}<0,05)$ na flexibilidade, na força de preensão manual e na força/resistência abdominal quando comparado aos valores iniciais $(\mathrm{p}<0,05)$.

\section{Discussão}

O tratamento multiprofissional para a obesidade severa tem foco na mudança do estilo de vida, com base na alteração dos hábitos alimentares e de atividade física, além do controle do estresse, sendo assim a base do tratamento (Abeso, 2016). Frente a esse problema, Biachini et al. (2016), Goodpaster et al. (2010), defendem a participação das pessoas em programas multiprofissionais de tratamento da obesidade, com base na adequação da realização de atividade física e hábitos alimentares, como também o controle do estresse e o aconselhamento psicológico (Bianchini et al., 2016; Goodpaster et al., 2010).

Após 24 semanas de intervenção os resultados evidenciaram que o Programa Multiprofissional de Tratamento da Obesidade (PMTO) promoveu melhoras significativas no IMC; na composição corporal: Percentual de Gordura e Massa de Gordura. Houve melhora também na aptidão física: na flexibilidade na força de preensão manual e na resistência muscular abdominal.

A redução significativa no IMC, também foi verificada em um estudo que analisou o efeito de um treinamento físico aquático (TFA) na aptidão física e capacidade funcional de mulheres com obesidade graus II e III, com média de 34,2 $\pm 8,3$ anos e IMC de 44,76 $\pm 10,08 \mathrm{~kg} / \mathrm{m}^{2}$ que fizeram TFA de 12 semanas. As mesmas apresentaram redução significativa na massa corporal, IMC e circunferência da cintura (Zaniboni et al., 2019).

Com relação à aptidão física, o PMTO proporcionou melhoria na flexibilidade, na força muscular de membros superiores, na resistência muscular dinâmica de membros inferiores e estática foram observadas evidências de aumento nos resultados semelhante ao que aconteceu nos estudos de Rikli e Jones (2013) e Chase et al. (2014).

No estudo de Westphal et al (2020), em mulheres com obesidade foi avaliado o treinamento funcional no período de 12 semanas, demonstrando melhorias nas variáveis antropométricas e de aptidão física relacionado à saúde (Westphal et al., 2020). O mesmo aconteceu no presente estudo, não houve melhoria no perfil lipídico, mas nas variáveis de composição corporal e de aptidão física houve melhorias, promovendo a autonomia destas pessoas, principalmente na capacidade funcional, elevando assim o seu nível de qualidade de vida.

Em um estudo conduzido por Goodpaster et al. (2010) para determinar a eficácia de uma intervenção para perda de peso e redução do risco cardiometabólico na obesidade grave, realizado na Universidade de Pittsburgh, com 130 participantes 
com obesidade grave (classe II ou III). A Intervenção intensiva de estilo de vida teve duração de um ano e consistia em dieta e atividade física. Para tanto, um grupo realizou atividade física desde o início do programa, juntamente com a dieta proposta, enquanto o outro grupo teve a intervenção dietética idêntica, mas com atividade física iniciada apenas após 6 meses de intervenção. Os resultados foram mudanças no peso e em desfechos secundários que eram componentes adicionais que indicam o risco cardiometabólico, incluindo circunferência da cintura, tecido adiposo abdominal e conteúdo de gordura hepática (Goodpaster et al., 2010). Estes achados assemelham-se com os do presente estudo.

De acordo com Swift et al. (2014) a participação em um programa de treinamento físico no tratamento da obesidade é importante para manter o controle da massa corporal a longo prazo (Swift et al., 2014). Nesta pesquisa a proposta de intervenções intensivas com exercícios em ambiente aquático, em comunhão com a orientação nutricional e psicológica demonstrou resultados significativos na diminuição de gordura e melhora na aptidão física relacionada à saúde.

O PMTO promoveu melhorias significativas em vários parâmetros estudados. No entanto, 24 semanas de intervenção não foram suficientes para promover mudanças mais expressivas no estado nutricional. Assim, indica que embora o programa seja efetivo na promoção de importantes melhorias à saúde, há a necessidade de investigação de outros parâmetros tais como um tempo maior de intervenção, diferentes orientações dietéticas e de mudança de comportamento, entre outras medidas. Apesar disso, estas limitações não são vistas de forma negativa, uma vez que o PMTO atendeu pessoas que necessitam de uma atenção qualificada e de longo prazo, que atualmente não está presente no sistema único de saúde (SUS). Com isso, os resultados apresentados servem de parâmetro para comparação com outros estudos semelhantes e também para a análise e implementação de intervenções congêneres em programas de saúde pública no contexto do SUS.

\section{Conclusão}

O programa de intervenção com exercícios em ambiente aquático, orientação para a adoção de uma dieta balanceada e suporte psicológico, promoveu perda de peso clinicamente significativa e melhorias na aptidão física em participantes com obesidade severa. Portanto, mostrou-se seguro e promissor para a realização de mais estudos com essa população como forma de ampliar o entendimento e sobre outros tipos de opções terapêutica disponíveis, sobretudo no âmbito do sistema único de saúde.

\section{Referências}

Abeso. (2016). Associação Brasileira para o Estudo da Obesidade e da Síndrome Metabólica. In Diretrizes brasileiras de obesidade (Número 4 ED.).

ACSM, A. C. of S. M. (2014). Diretrizes do ACSM para os Testes de Esforço e sua Prescrição.

Alberton, C. L., \& Kruel, L. F. M. (2009). Influência da imersão nas respostas cardiorrespiratórias em repouso. Revista Brasileira de Medicina do Esporte, 15(3), 228-232. https://doi.org/10.1590/S1517-86922009000300013

Andy Field. (2009). Discovering Statistics using SPSS Statistics. SAGE Publications, 66, 822. http://www.amazon.com/Discovering-Statistics-using-IBMSPSS/dp/1446249182

Associação Brasileira para o Estudo da Obesidade e Síndrome Metabólica, A. (2020). Obesidade e sobrepeso. https://abeso.org.br/conceitos/obesidade-esobrepeso/

ATS. (2002). American Thoracic Society ATS Statement: Guidelines for the Six-Minute Walk Test. American journal of respiratory and critical care medicine, 166, 111-117. https://doi.org/10.1164/rccm.166/1/111

Bevilaqua, C. A., Pelloso, S. M., \& Marcon, S. S. (2016). Estágio de mudança de comportamento em mulheres de um programa multiprofissional de tratamento da obesidade. Revista Latino-Americana de Enfermagem, 24(0). https://doi.org/10.1590/1518-8345.0549.2809

Bianchini, J. A. A., Silva, D. F. Da, Lopera, C. A., Antonini, V. D. S., \& Nardo Junior, N. (2016). Intervenção multiprofissional melhora a aptidão física relacionada à saúde de adolescentes com maior efeito sobre as meninas em comparação aos meninos. Revista Brasileira de Educação Física e Esporte, 30(4), 1051-1059. https://doi.org/10.1590/1807-55092016000401051

Bianchini, J. A. A., Silva, D. F. Da, Lopera, C. A., Antonini, V. D. S., \& Nardo Junior, N. (2016). Intervenção multiprofissional melhora a aptidão física relacionada à saúde de adolescentes com maior efeito sobre as meninas em comparação aos meninos. Revista Brasileira de Educação Física e Esporte, 30(4), 
1051-1059. https://doi.org/10.1590/1807-55092016000401051

Borg, G. A. V. (1982). Psychophysical bases of perceived exertion”, Medicine and Science in Sports and Exercice, 1982. 377-381.

Brasil, M. S. (2019). Vigitel Brasil 2018: Vigilância de fatores de risco e proteção para doenças crônicas por inquerito telefônico. In G. Estatística e Informação em Saúde. http://bvsms.saude.gov.br/bvs/publicacoes/vigitel_brasil_2011_fatores_risco_doencas_cronicas.pdf

Brasil, V. (2016). Vigilância De Fatores De Risco E Proteção Para Dcnt. https://www.ans.gov.br/images/Vigitel_Saude_Suplementar.pdf

Caputo, E. L., Da Silva, M. C., \& Rombaldi, A. J. (2014). ComparaÇÃo entre diferentes protocolos de medida de forÇa de preens ̃̃o manual. Revista da Educacao Fisica, 25(3), 481-487. https://doi.org/10.4025/reveducfis.v25i3.23709

Cardoso, J., Azevedo, N., Cassano, C., Kawano, M., \& Âmbar, G. (2007). Confiabilidade intra e interobservador da análise cinemática angular do quadril durante o teste sentar e alcançar para mensurar o comprimento dos isquiotibiais em estudantes universitários. Revista Brasileira de Fisioterapia, 11(2), 133138. https://doi.org/10.1590/s1413-35552007000200008

Chase, K. ., C.E., B., FACSM, J. P., \& Coste, S. (2014). Fitness Norms for the Plank Exercise. International Journal of Exercise Science: Conference Proceedings, $8(2)$. https://digitalcommons.wku.edu/ijesab/vol8/iss2/14

Di Cesare, M., Bentham, J., Stevens, G. A., Zhou, B., Danaei, G., Lu, Y., Bixby, H., Cowan, M. J., Riley, L. M., Hajifathalian, K., Fortunato, L., Tad dei, C., Bennett, J. E., Ikeda, N., Khang, Y. H., Kyobutungi, C., Laxmaiah, A., Li, Y., Lin, H. H., ... Cisneros, J. Z. (2016a). Trends in adult body-mass index in 200 countries from 1975 to 2014: A pooled analysis of 1698 population-based measurement studies with 19.2 million participants. The Lancet, 387(10026), 13771396. https://doi.org/10.1016/S0140-6736(16)30054-X

Di Cesare, M., Bentham, J., Stevens, G. A., Zhou, B., Danaei, G., Lu, Y., Bixby, H., Cowan, M. J., Riley, L. M., Hajifathalian, K., Fortunato, L., Taddei, C., Bennett, J. E., Ikeda, N., Khang, Y. H., Kyobutungi, C., Laxmaiah, A., Li, Y., Lin, H. H., ... Cisneros, J. Z. (2016b). Trends in adult body-mass index in 200 countries from 1975 to 2014: A pooled analysis of 1698 population-based measurement studies with 19.2 million participants. The Lancet, 387(10026), 13771396. https://doi.org/10.1016/S0140-6736(16)30054-X

Dobbs, R., C, S., F, T., J, M., JR, W., P, C., S, M., \& A., S. (2014). Overcoming obesity: an initial economic analysis. McKinsey Global Institute.

Egídio, J. P. de O., Foss-Freitas, M. C., Junior, R. M. M., \& Vencio, S. (2017). Diretrizes da Sociedade Brasileira de Diabetes 2017-2018. In Clannad. https://doi.org/10.5935/abc.20170188

Faludi, Izar, Saraiva, APM, C., Bianco HT, \& A, A. N. (2017). Atualização da Diretriz Brasileira de Dislipidemias e Prevenção da Aterosclerose - 2017. Arquivos Brasileiros de Cardiologia, 109(2Supl.1), 76. https://doi.org/10.5935/abc.2013S010

Goodpaster, B., DeLany, J. P., Otto, A. D., Kuller, L., Vockley, J., Jeannette E. South-Paul, S. B. T., Brown, J., McTigu, K., Hames, K. C., Lang, W., \& Jakicic, J. M. (2010). Effects of Diet and PA intervention on WT loss and Cardiometabolic risk factor in very obese adults. Jama, 304(16), 1795-1802. https://doi.org/10.1001/jama.2010.1505.Effects

Jensen, M. D., Ryan, D. H., Apovian, C. M., Ard, J. D., Comuzzie, A. G., Donato, K. A., Hu, F. B., Hubbard, V. S., Jakicic, J. M., Kushner, R. F., Loria, C. M., Millen, B. E., Nonas, C. A., Pi-Sunyer, F. X., Stevens, J., Stevens, V. J., Wadden, T. A., Wolfe, B. M., \& Yanovski, S. Z. (2014). 2013 AHA/ACC/TOS guideline for the management of overweight and obesity in adults: A report of the American college of cardiology/American heart association task force on practice guidelines and the obesity society. Journal of the American College of Cardiology, 63(25 PART B), $2985-3023$. https://doi.org/10.1016/j.jacc.2013.11.004

Jones, C. J., \& Rikli, R. (2012). Development and Validation of Criterion-Referenced, Clinically Relevant Fitness Standards for Maintaining Physical Independence in Later Years. Gerontologist, 52, 343-344.

Lau, D. C. W., Douketis, J. D., Morrison, K. M., Hramiak, I. M., Sharma, A. M., \& Ur, E. (2007). 2006 Canadian clinical practice guidelines on the management and prevention of obesity in adults and children [summary]. CMAJ : Canadian Medical Association journal = journal de l'Association medicale canadienne, 176(8). https://doi.org/10.1503/cmaj.061409

Lopera, C. A., da Silva, D. F., Bianchini, J. A. A., Locateli, J. C., Moreira, A. C. T., Dada, R. P., Thivel, D., \& Nardo, N. (2016). Effect of water- versus landbased exercise training as a component of a multidisciplinary intervention program for overweight and obese adolescents. Physiology and Behavior, $165,365-$ 373. https://doi.org/10.1016/j.physbeh.2016.08.019

Nardo Junior, N., Bianchini, J. A. A., da Silva, D. F., Ferraro, Z. M., Lopera, C. A., \& Antonini, V. D. S. (2018). Building a response criterion for pediatric multidisciplinary obesity intervention success based on combined benefits. European Journal of Pediatrics, 177(6). https://doi.org/10.1007/s00431-018-31150

Pataky, Z., Armand, S., Müller-Pinget, S., Golay, A., \& Allet, L. (2014). Effects of obesity on functional capacity. Obesity, 22(1), 56-62. https://doi.org/10.1002/oby.20514

Patsopoulos, N. A. (2011). A pragmatic view on pragmatic trials. Dialogues in Clinical Neuroscience, 13(2), 217-224.

Stegenga, H., Haines, A., Jones, K., \& Professor, J. W. (2014). Identification, assessment, and management of overweight and obesity: Summary of updated nice guidance. The BMJ, 349. https://doi.org/10.1136/bmj.g6608

Swift, D. L., Johannsen, N. M., Lavie, C. J., Earnest, C. P., \& Church, T. S. (2014). The role of exercise and physical activity in weight loss and maintenance. Progress in Cardiovascular Diseases, 56(4), 441-447. https://doi.org/10.1016/j.pcad.2013.09.012

Westphal, G., Baruki, S. B. S., Mori, T. A. de, Montebello, M. I. de L., \& Pazzianotto-Forti, E. M. (2020). Effects of Individualized Functional Training on the Physical Fitness of Women with Obesity. Lecturas: Educación Física y Deportes, 25(268), 61-75. https://doi.org/10.46642/efd.v25i268.2084

Withrow, D., \& Alter, D. A. (2011). The economic burden of obesity worldwide: A systematic review of the direct costs of obesity. Obesity Reviews, 12(2), 
Research, Society and Development, v. 10, n. 1, e12910111636, 2021

(CC BY 4.0) | ISSN 2525-3409 | DOI: http://dx.doi.org/10.33448/rsd-v10i1.11636

131-141. https://doi.org/10.1111/j.1467-789X.2009.00712.x

World Health Organization, W. (2011). WHO | Waist Circumference and Waist-Hip Ratio. Report of a WHO Expert Consultation. Geneva, 8-11 December 2008. December, 8-11. http://www.who.int

Zaniboni, G. R., Baruki, S. B. S., Cesar, M. de C., Rasera Júnior, I., \& Pazzianotto-Forti, E. M. (2019). Treinamento físico aquático melhora capacidade funcional e aptidão física em mulheres com obesidade graus II e III. Revista Brasileira de Ciências do Esporte, 41(3), 314-321. https://doi.org/10.1016/j.rbce.2018.06.007 